\title{
Comparison and Enhancement of Digital Image by Using Canny Filter and Sobel Filter
}

\author{
Rupika Rana ${ }^{1}$, Ashish Verma $^{2}$ \\ ${ }^{1,2}$ (Computer Science \& Engineering, SSIET College/ PTU, India)
}

\begin{abstract}
In this research paper we have defining two different edge detection methods i.e canny edge detection and Sobel edge detection and we are also discussing some image quality parameters like PSNR, SNR, MSE, RMSE, UQI and MAE. Edge detection is a technique that is used to identifying points in a digital image at which the image brightness changes sharply or, more formally, has discontinuities. The points at which image brightness changes sharply are typically organized into a set of curved line segments termed edges. Results shows that our strategy on the nature of comparison between canny and sobel edge detection methods by using image quality parameters on Medical images [MRI and CT Scan] and to further finding a better and more accurate edge detection method.The values of these methods are interpreted and illustrated in results section .

Keywords: Canny, Sobel, Edge Detection, Medical Images, Quality Measurement, MRI, CT scan.
\end{abstract}

\subsection{Edge Detection:}

\section{INTRODUCTION}

Edge detection is a technique in which the points where image brightness changes sharply or formally are identified. These points are organized under line segments called edges. Edge detection also aims to classify and place discontinuities in an image [2].

\subsection{Edge properties}

The edges extracted from a two-dimensional image of a three-dimensional scene can be classified as either viewpoint dependent or viewpoint independent. A typical edge might for instance be the border between a block of red color and a block of yellow. In contrast a line (as can be extracted by a ridge detector) can be a small number of pixels of a different color on an otherwise unchanging background. For a line, there may therefore usually be one edge on each side of the line. [4]

\subsubsection{Canny Edge Detection}

The Canny edge detector is an edge detection operator that uses a multi-stage algorithm to detect a wide range of edges in images. It was developed by John F. Canny in 1986. Canny also produced a computational theory of edge detection explaining why the technique works. Canny's aim was to discover the optimal edge detection algorithm. [5]

\subsubsection{Sobel Edge Detection}

The Sobel operator is used for edge detection algorithms. It is a discrete differentiation operator, computing an approximation of the gradient of the image intensity function. [3] At each point in the image, the result of the Sobel operator is either the corresponding gradient vector or the norm of this vector. The Sobel operator is based on convolving the image with a small, separable, and integer valued filter in horizontal and vertical direction and is therefore relatively inexpensive in terms of computations. On the other hand, the gradient approximation that it produces is relatively crude, in particular for high frequency variations in the image. [8]

The Sobel operator consists of two separable operations:

- Smoothing perpendicular to the derivative direction with a triangle filter.

- Simple central difference in the derivative direction.

\section{RESEARCH GAP}

Literature survey has revealed only two studies which have attempted to predict the high and low values of image quality parameters.

- Edge detection algorithms provide directional information for the analysis of an image. Medical images such as Magnetic Resonance Imaging (MRI) and Computed Tomography (CT Scan) are nearly the main examination tool for medical examination because they have wealthy information about the condition of anatomical structures and biological tissues. 
- According to the literature survey, various researchers offered different edge detection algorithms for different types of images i.e. images of wood decay, fire, medical, facial image and binocular images etc. Some researchers proposed enhanced versions of popular edge detection algorithms such as gabor, canny, sobel \& prewitt etc. [9] Each algorithm has its own merits and demerits and researchers are trying to overcome from all these by comparing their results with each other but no one has analyzed the image quality on the basis of image quality parameters such as PSNR, SNR, MSE, RMSE, MAE, UIQ etc.

\section{IMPLEMENTATION STEPS}

The entire study was conducted in a following manner as listed below:

$\begin{array}{ll}\text { 1) Input image I } & \text { 2) Smoothing (I) for noise removal. }\end{array}$

4) Non-max suppression

5) Double thresholding

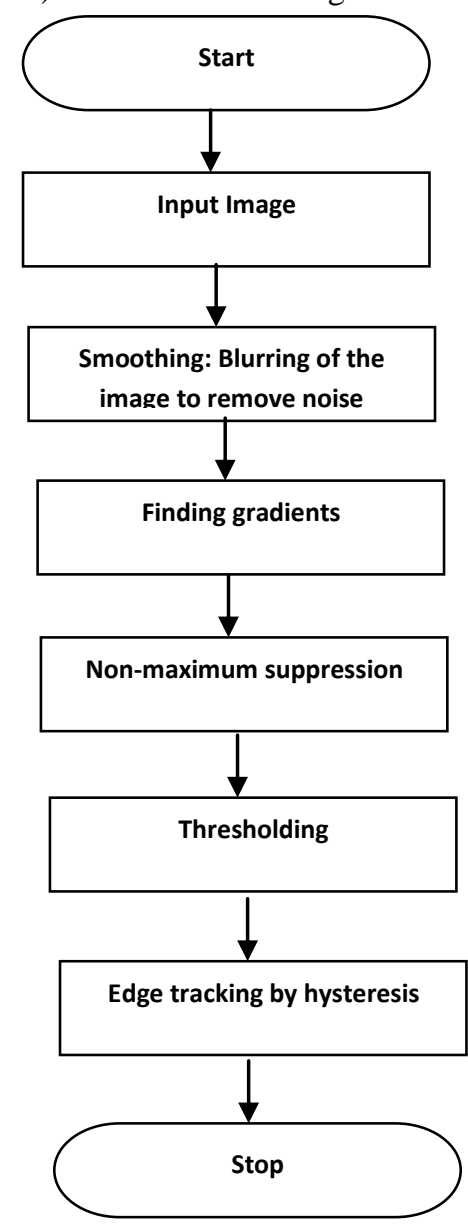

3) Find gradients (I)

6) Edge tracking by hysteresis

Figure 1: Flow Chart of Proposed Algorithm

STEP 1: Input Image (I)

STEP 2: Smoothing (I) for noise removal.

STEP 3: Find gradients (I):

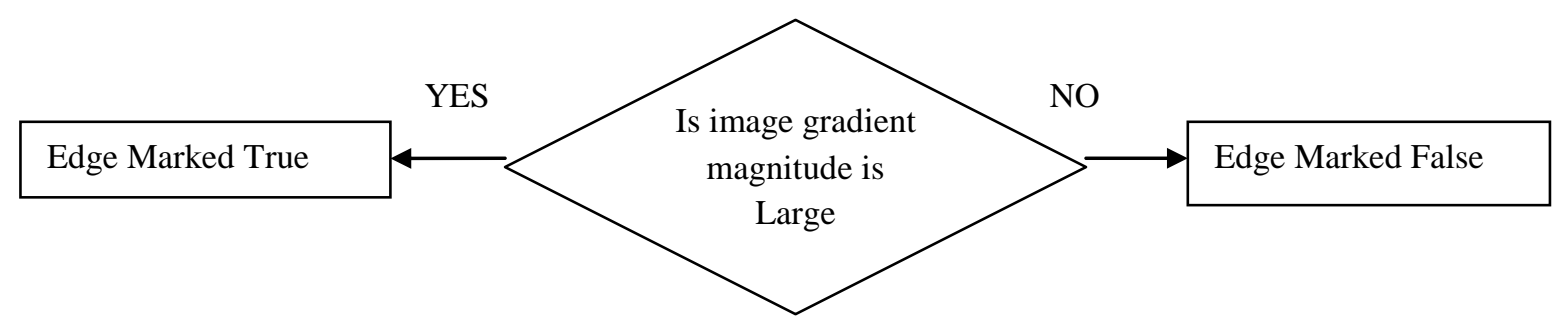

Figure 2: Flow Chart for finding gradients of Image (I) 


Table for the above flowchart is shown below:
\begin{tabular}{|c|c|c|}
\hline Image Name & Gradients Magnitude value & Edge Marked \\
\hline I & LARGE & TRUE \\
\hline I & SMALL & FALSE \\
\hline
\end{tabular}

STEP 4: Non-maximum suppression: After finding image gradients magnitude value is large and lies in local maxima then it will be marked as an edge.

STEP 5: Double thresholding: Potential edges are determined by thresholding. Large intensity gradients are more likely to correspond to edges than small intensity gradients.

STEP 6: Edge tracking by hysteresis: It is in most cases impossible to specify a threshold at which a given intensity gradient switches from corresponding to an edge. Therefore Canny uses thresholding with hysteresis. For each edge $\mathrm{E}$ in I If $\mathrm{E}$ is not connected to a very certain (strong) edge then suppress E.[1]

\subsection{TABLE}

Following table shows Canny edge detection and Sobel edge detection algorithm and different parameters like PSNR, SNR, MSE, RMSE, UQI and MAE etc. Canny method can produce good edge with the smooth pixels whereas Sobel edge detection method cannot produce smooth edge compared to canny method of Medical images(CT Scan \& MRI Scan).

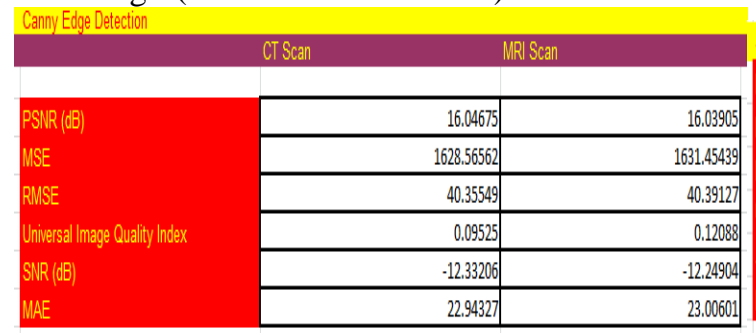

\begin{tabular}{|c|c|c|}
\hline Sobel Edge Detection & & \\
\hline$P S \| R(A B)$ & 16,03962 & 16,02715 \\
\hline MSE & 1631.23989 & 1635,93264 \\
\hline RMSE & 40,38861 & 40,46666 \\
\hline Universal Image: Quality Index & 0.14757 & 0.12804 \\
\hline $\operatorname{SNR}(\mathbb{B})$ & $-12,30613$ & -11.86198 \\
\hline MAE & 23.1365 & $23: 11414$ \\
\hline
\end{tabular}

Table 1: Performance Analysis Table for Canny \& Sobel edge detection.

\section{Results}

4.1 GRAPHS: The following graphs are depicted from the above performance analysis table values.
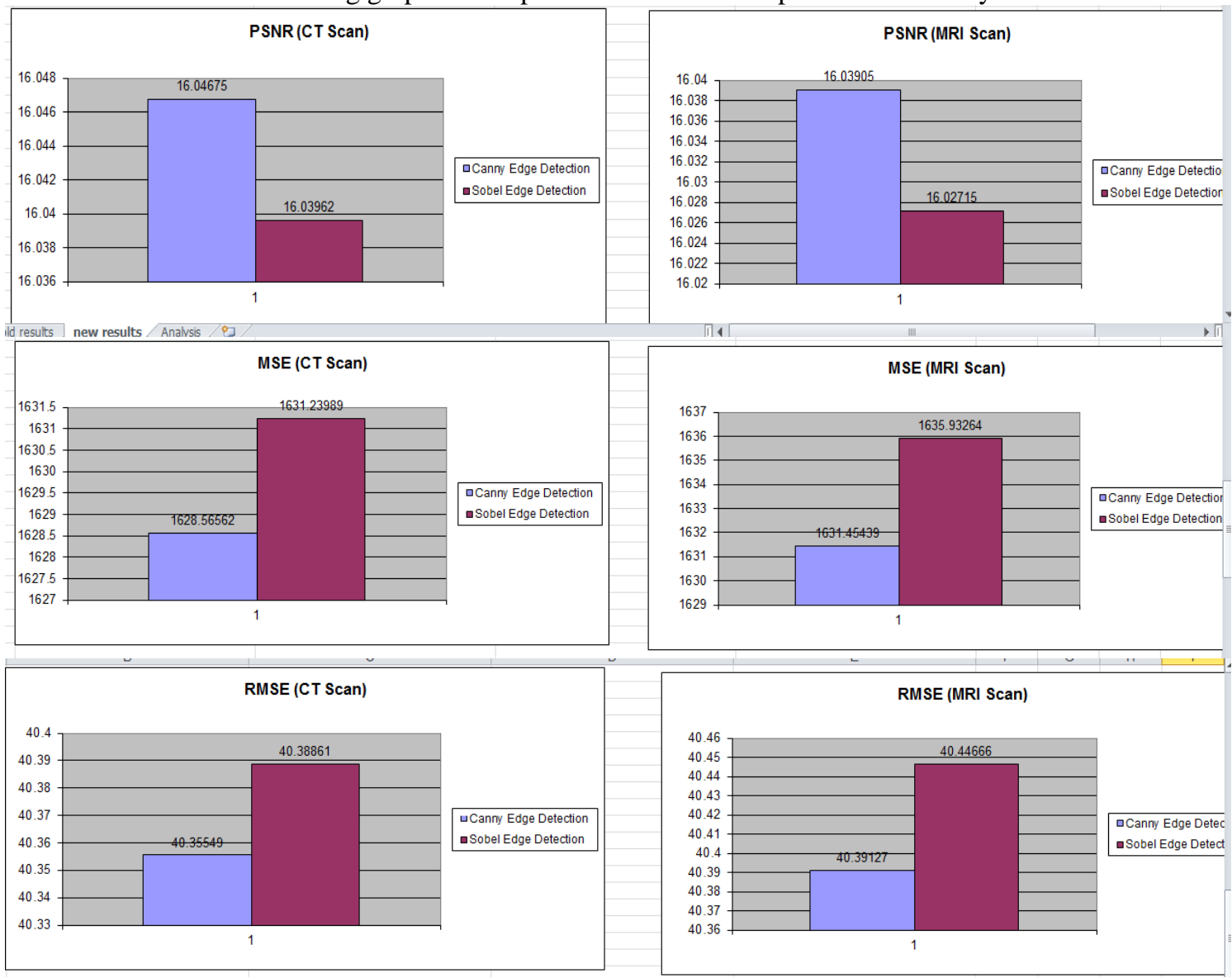

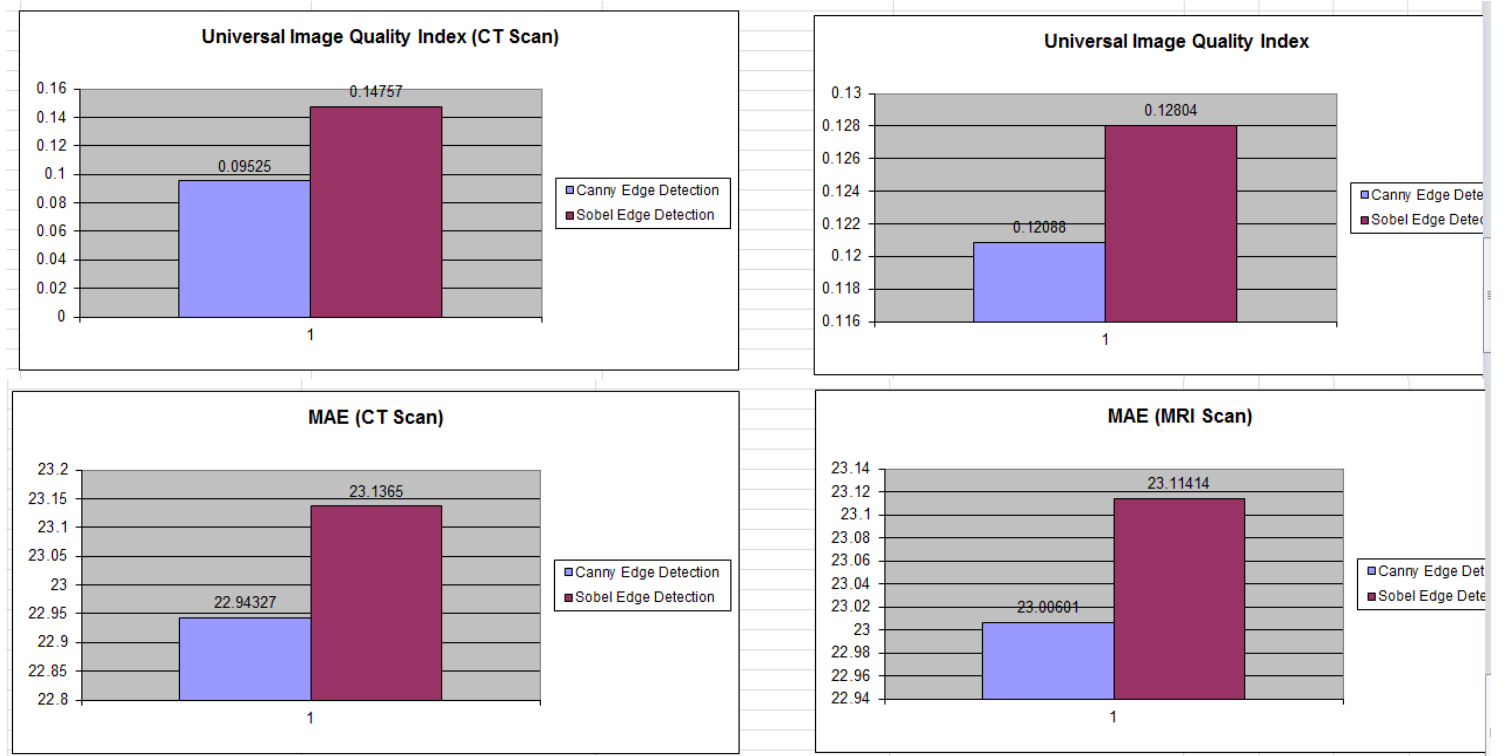

4.2 Result Images in MATLAB: This research work includes the performance analysis of two different edge detection algorithms i.e. canny edge detection and Sobel edge detection algorithm. We used different images of CT scan and MRI Scan for analysis purpose. Finally we compared the image quality using different parameters like PSNR, SNR, MSE, RMSE, UQI and MAE etc as shown in following resultant images.

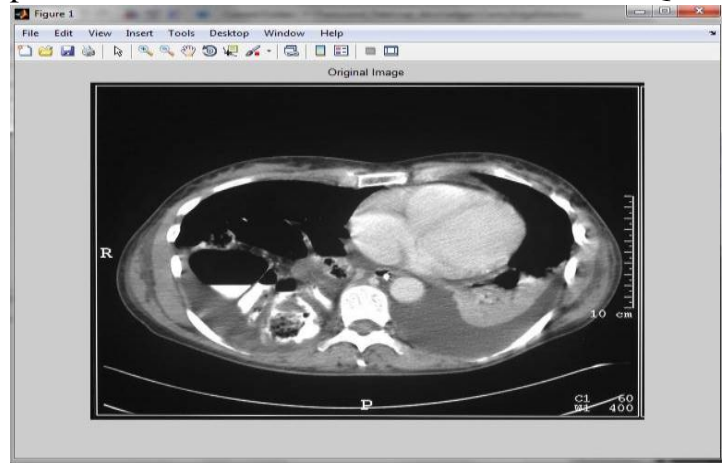

Input Image 1: CT Scan

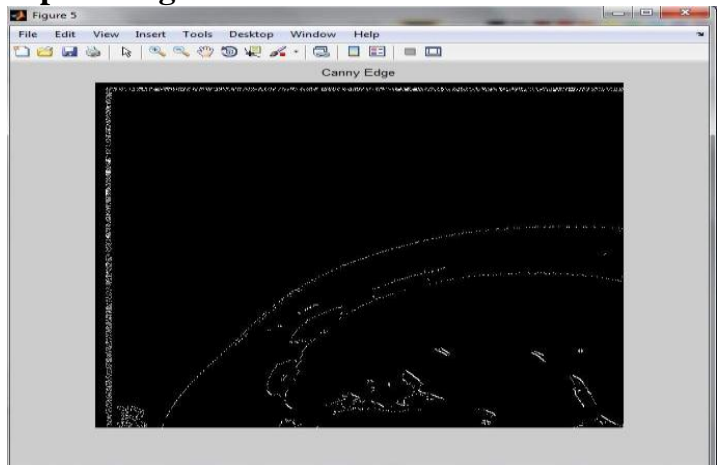

Figure 3: Final Result of CT Scan by Using Canny Detection.

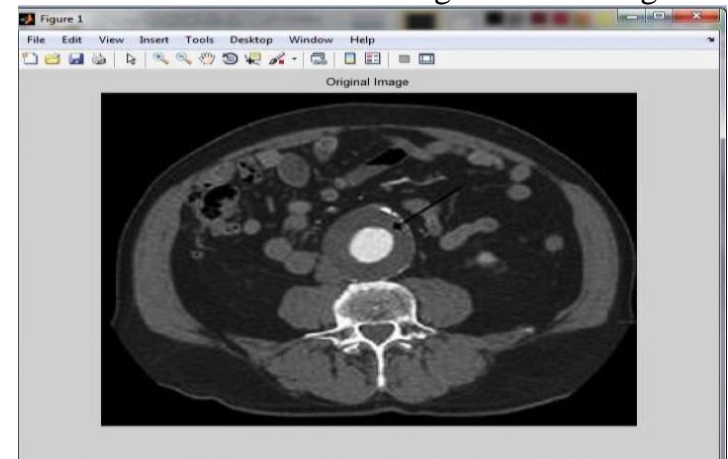

\section{Input Image 2: MRI Scan}

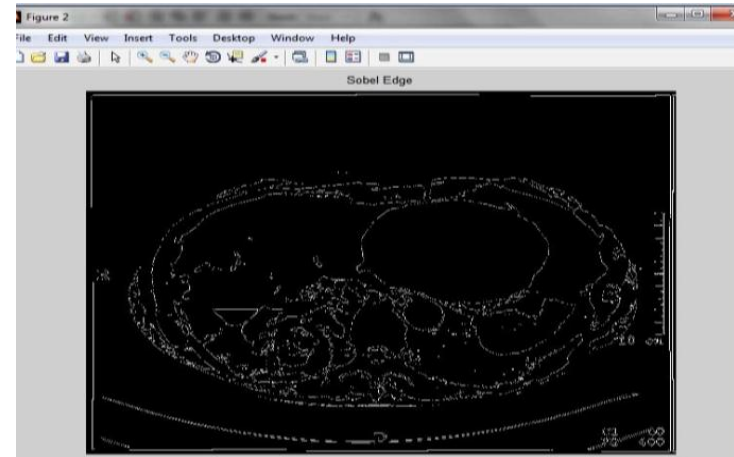

Figure 4: Final Result of CT Scan

By Using Sobel Edge Detection. 


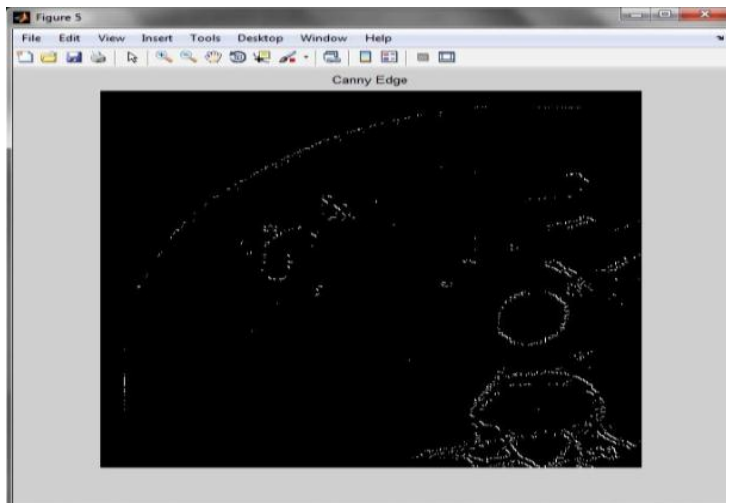

Figure 5: Final Result of MRI Scan by Using Canny Edge Detection.

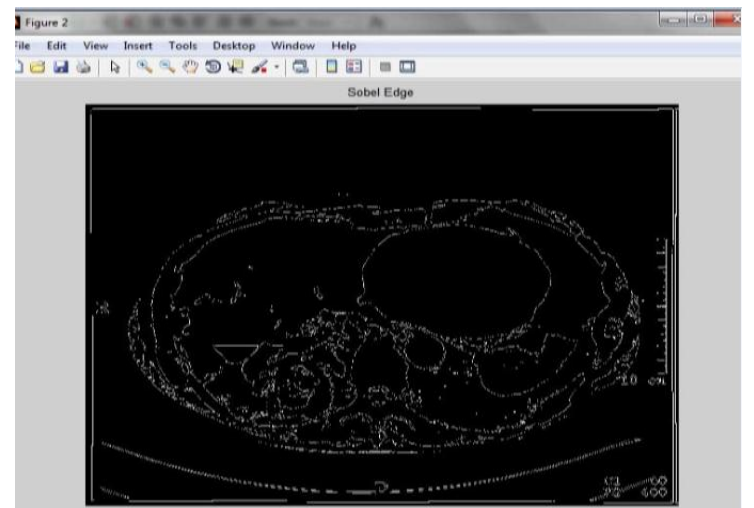

Figure 6: Final Result of MRI Scan by Using Sobel Edge Detection.

\section{Conclusion}

In this paper, we explored two different edge detection algorithms i.e Canny edge detection and Sobel edge detection algorithm and different parameters like PSNR, SNR, MSE, RMSE, UQI and MAE etc. Canny method can produce good edge with the smooth continuous pixels and thin edge. Sobel edge detection method cannot produce smooth and thin edge compared to canny method. From our analysis, we have shown that between Sobel and Canny edge detection algorithms, response given by canny edge detection is better than result of Sobel detector used in CT Scan and MRI images.

\section{References}

[1] Rafael C. Gonzalez, Richard Eugene Woods, "Digital image processing"

[2] Muthukrishnan.R and M.Radha of Edge Detection Techniques for image Segmentation, International Journal of Computer Science \& Information Technology (IJCSIT), Dec 2011.

[3] A Study on Image Edge Detection Using the Gradients, International Journal of Scientific and Research Publications, December 2012 .

[4] A Brief, Early History of Computer Graphics in Film (Larry Yeager, 16 August 2002 (last update), retrieved 24 March 2010).

[5] Edge Xiangjian, Jianmin Li, Darning wer', Wenjing Jia 2 and Qiang Wu2, Canny Detection on a Virtual Hexagonal Image Structure.

[6] Elgammal, Ahmed, CS 534: Computer Vision 3D Model-based recognition,(Dept. of Computer Science, Rutgers University).

[7] Liu Hong-Ming, Study and Comparison of Various Image Edge Detection Techniques, Science \& Technology Information, No. 27, 2009.

[8] I. Sobel, Neighborhood coding of binary images fast contour following and general array binary processing, Computer Graphics and Image Processing 8 (1978) 127-135

[9] Zolqernine Othman, Comparison of Canny and Sobel Edge Detection in MRI Images. 